\title{
Approximation by trigonometric polynomials in weighted Orlicz spaces
}

\author{
by \\ Daniyal M. Israfilov and Ali Guven (Balikesir)
}

\begin{abstract}
We investigate the approximation properties of the partial sums of the Fourier series and prove some direct and inverse theorems for approximation by polynomials in weighted Orlicz spaces. In particular we obtain a constructive characterization of the generalized Lipschitz classes in these spaces.
\end{abstract}

1. Introduction and main results. A convex and continuous function $M:[0, \infty) \rightarrow[0, \infty)$ for which $M(0)=0, M(x)>0$ for $x>0$ and

$$
\lim _{x \rightarrow 0} \frac{M(x)}{x}=0, \quad \lim _{x \rightarrow \infty} \frac{M(x)}{x}=\infty
$$

is called a Young function. The complementary Young function $N$ of $M$ is defined by

$$
N(y):=\max \{x y-M(x): x \geq 0\}
$$

for $y \geq 0$.

Let $\mathbb{T}$ denote the interval $[-\pi, \pi], \mathbb{C}$ the complex plane, and $L_{p}(\mathbb{T}), 1 \leq$ $p \leq \infty$, the Lebesgue space of measurable complex-valued functions on $\mathbb{T}$.

For a given Young function $M$ let $\widetilde{L}_{M}(\mathbb{T})$ denote the set of all Lebesgue measurable functions $f: \mathbb{T} \rightarrow \mathbb{C}$ for which

$$
\int_{\mathbb{T}} M(|f(x)|) d x<\infty .
$$

The linear span of $\widetilde{L}_{M}(\mathbb{T})$ equipped with the Orlicz norm

$$
\|f\|_{L_{M}(\mathbb{T})}:=\sup \left\{\int_{\mathbb{T}}|f(x) g(x)| d x: g \in \widetilde{L}_{N}(\mathbb{T}), \int_{\mathbb{T}} N(|g(x)|) d x \leq 1\right\},
$$

where $N$ is the complementary Young function of $M$, or with the Luxemburg

2000 Mathematics Subject Classification: 41A25, 41A27, 42A10, 46E30.

Key words and phrases: Orlicz space, weighted Orlicz space, Boyd indices, modulus of smoothness, Muckenhoupt class, direct theorems, inverse theorems, best approximation. 
norm

$$
\|f\|_{L_{M}(\mathbb{T})}^{*}:=\inf \left\{\lambda>0: \int_{\mathbb{T}} M\left(\frac{|f(x)|}{\lambda}\right) d x \leq 1\right\}
$$

becomes a Banach space. This space is denoted by $L_{M}(\mathbb{T})$ and is called an Orlicz space [26, p. 69]. The Orlicz and Luxemburg norms satisfy [26, p. 80] the inequalities

$$
\|f\|_{L_{M}(\mathbb{T})}^{*} \leq\|f\|_{L_{M}(\mathbb{T})} \leq 2\|f\|_{L_{M}(\mathbb{T})}^{*}, \quad f \in L_{M}(\mathbb{T}),
$$

and hence they are equivalent. Furthermore, the Orlicz norm can be determined by means of the Luxemburg norm [26, pp. 79-80]:

$$
\|f\|_{L_{M}(\mathbb{T})}:=\sup \left\{\int_{\mathbb{T}}|f(x) g(x)| d x:\|g\|_{L_{N}(\mathbb{T})}^{*} \leq 1\right\}
$$

and then the Hölder inequalities

$$
\begin{aligned}
& \int_{\mathbb{T}}|f(x) g(x)| d x \leq\|f\|_{L_{M}(\mathbb{T})}\|g\|_{L_{N}(\mathbb{T})}^{*}, \\
& \int_{\mathbb{T}}|f(x) g(x)| d x \leq\|f\|_{L_{M}(\mathbb{T})}^{*}\|g\|_{L_{N}(\mathbb{T})}
\end{aligned}
$$

hold for every $f \in L_{M}(\mathbb{T})$ and $g \in L_{N}(\mathbb{T})[26$, p. 80].

W. Matuszewska and W. Orlicz [32] have associated a pair of indices with a given Orlicz space $L_{M}(\mathbb{T})$. A generalization of these, or rather their reciprocals, has been given in the more general context of rearrangement invariant spaces in [5]. Let $M^{-1}:[0, \infty) \rightarrow[0, \infty)$ be the inverse of the Young function $M$ and let

$$
h(t):=\limsup _{x \rightarrow \infty} \frac{M^{-1}(x)}{M^{-1}(t x)}, \quad t>0 .
$$

The numbers $\alpha_{M}$ and $\beta_{M}$ defined by

$$
\alpha_{M}:=\lim _{t \rightarrow \infty}-\frac{\log h(t)}{\log t}, \quad \beta_{M}:=\lim _{t \rightarrow 0^{+}}-\frac{\log h(t)}{\log t}
$$

are called the lower and upper Boyd indices of the Orlicz space $L_{M}(\mathbb{T})$ respectively. It is known that

$$
0 \leq \alpha_{M} \leq \beta_{M} \leq 1
$$

and

$$
\alpha_{N}+\beta_{M}=1, \quad \alpha_{M}+\beta_{N}=1 .
$$

The Orlicz space $L_{M}(\mathbb{T})$ is reflexive if and only if $0<\alpha_{M} \leq \beta_{M}<1$, i.e. if the Boyd indices are nontrivial.

If $1 \leq q<1 / \beta_{M} \leq 1 / \alpha_{M}<p \leq \infty$, then $L_{p}(\mathbb{T}) \subset L_{M}(\mathbb{T}) \subset L_{q}(\mathbb{T})$, the inclusions being continuous, and hence $L_{\infty}(\mathbb{T}) \subset L_{M}(\mathbb{T}) \subset L_{1}(\mathbb{T})$. The above information concerning the Boyd indices can be found in [3, p. 350]. 
We refer to [2], [4]-[6] and [28] for a complete discussion of Boyd indices properties.

A measurable function $\omega: \mathbb{T} \rightarrow[0, \infty]$ is called a weight function if the set $\omega^{-1}(\{0, \infty\})$ has Lebesgue measure zero.

Let $\omega$ be a weight function. We denote by $L_{M}(\mathbb{T}, \omega)$ the linear space of all measurable functions $f$ such that $f \omega \in L_{M}(\mathbb{T})$ and set

$$
\|f\|_{L_{M}(\mathbb{T}, \omega)}:=\|f \omega\|_{L_{M}(\mathbb{T})} .
$$

The normed space $L_{M}(\mathbb{T}, \omega)$ is called a weighted Orlicz space.

From the Hölder inequality it follows that if $\omega \in L_{M}(\mathbb{T})$ and $1 / \omega \in$ $L_{N}(\mathbb{T})$, then $L_{\infty}(\mathbb{T}) \subset L_{M}(\mathbb{T}, \omega) \subset L_{1}(\mathbb{T})$.

Let $1<p<\infty$ and $1 / p+1 / q=1$. A weight function $\omega$ belongs to the Muckenhoupt class $A_{p}(\mathbb{T})$ if

$$
\left(\frac{1}{|J|} \int_{J} \omega^{p}(x) d x\right)^{1 / p}\left(\frac{1}{|J|} \int_{J} \omega^{-q}(x) d x\right)^{1 / q} \leq C
$$

with a finite constant $C$ independent of $J$, where $J$ is any subinterval of $\mathbb{T}$ and $|J|$ denotes the length of $J$.

Let $L_{M}(\mathbb{T}, \omega)$ be a weighted Orlicz space with Boyd indices $0<\alpha_{M} \leq$ $\beta_{M}<1$, and let $\omega \in A_{1 / \alpha_{M}}(\mathbb{T}) \cap A_{1 / \beta_{M}}(\mathbb{T})$. For $f \in L_{M}(\mathbb{T}, \omega)$ we define the shift operator $\sigma_{h}$ by

$$
\left(\sigma_{h} f\right)(x):=\frac{1}{2 h} \int_{-h}^{h} f(x+t) d t, \quad 0<h<\pi, x \in \mathbb{T},
$$

and the $k$-modulus of smoothness $\Omega_{M, \omega}^{k}(\cdot, f)(k=1,2, \ldots)$ by

$$
\Omega_{M, \omega}^{k}(\delta, f):=\sup _{\substack{0<h_{i} \leq \delta \\ 1 \leq i \leq k}}\left\|\prod_{i=1}^{k}\left(I-\sigma_{h_{i}}\right) f\right\|_{L_{M}(\mathbb{T}, \omega)}, \quad \delta>0,
$$

where $I$ is the identity operator. This modulus of smoothness is well defined, because we will prove (Lemma 1 ) that $\sigma_{h}$ is a bounded linear operator on $L_{M}(\mathbb{T}, \omega)$.

We define the shift operator $\sigma_{h}$ and the modulus of smoothness $\Omega_{M, \omega}^{k}$ in this way, because the space $L_{M}(\mathbb{T}, \omega)$ is not, in general, invariant under the usual shift $f(x) \mapsto f(x+h)$.

In the case of $k=0$ we set $\Omega_{M, \omega}^{0}(\delta, f):=\|f\|_{L_{M}(\mathbb{T}, \omega)}$ and if $k=1$ we write $\Omega_{M, \omega}(\delta, f):=\Omega_{M, \omega}^{1}(\delta, f)$. The modulus of smoothness $\Omega_{M, \omega}^{k}(\cdot, f)$ is a nondecreasing, nonnegative, continuous function and

$$
\Omega_{M, \omega}^{k}(\delta, f+g) \leq \Omega_{M, \omega}^{k}(\delta, f)+\Omega_{M, \omega}^{k}(\delta, g)
$$

for $f, g \in L_{M}(\mathbb{T}, \omega)$. 
We denote by $E_{n}(f)_{M, \omega}$ the best approximation of $f \in L_{M}(\mathbb{T}, \omega)$ by trigonometric polynomials of degree not exceeding $n$, i.e.,

$$
E_{n}(f)_{M, \omega}=\inf \left\{\left\|f-T_{n}\right\|_{L_{M}(\mathbb{T}, \omega)}: T_{n} \in \Pi_{n}\right\},
$$

where $\Pi_{n}$ denotes the class of trigonometric polynomials of degree at most $n$. Note that the existence of $T_{n}^{*} \in \Pi_{n}$ such that

$$
E_{n}(f)_{M, \omega}=\left\|f-T_{n}^{*}\right\|_{L_{M}(\mathbb{T}, \omega)}
$$

follows, for example, from Theorem 1.1 in [8, p. 59].

In the literature many results on such approximation problems have been obtained, in particular, direct and inverse theorems for approximation by trigonometric polynomials in weighted and nonweighted Lebesgue spaces. The elegant presentation of the corresponding result in the nonweighted Lebesgue spaces $L^{p}(\mathbb{T}), 1 \leq p \leq \infty$, can be found in [40] and [8]. The best approximation problem by trigonometric polynomials in weighted spaces with weights satisfying the so-called $A_{p}(\mathbb{T})$-condition was investigated in [16] and [27]. In particular, using the $L^{p}(\mathbb{T}, \omega)$ version of the $k$-modulus of smoothness $\Omega_{M, \omega}^{k}(\cdot, f), k=1,2, \ldots$, some direct and inverse theorems in weighted Lebesgue spaces were obtained in [16]. Generalizations of those results to weighted Lebesgue spaces defined on the curves of complex plane were proved in [19]-[21].

More general doubling weights, approximation by trigonometric polynomials in the periodic case and other related problems were studied in [29], [30], [31], [7]. Direct and converse results in the case of exponential weights on the real line were obtained in [14] and [15]. Some interesting results concerning best polynomial approximation in weighted Lebesgue spaces were also proved in [9] and [10]. Detailed information on weighted polynomial approximation can be found in the books [11] and [33]. Direct problems in nonweighted Orlicz spaces were studied in [36], [41] and [39]. To the best of the author's knowledge there are no results on approximation by trigonometric polynomials in weighted Orlicz spaces. In this work we prove some direct and inverse theorems of approximation theory in the weighted Orlicz spaces $L_{M}(\mathbb{T}, \omega)$. In particular, we obtain a constructive characterization of the generalized Lipschitz classes defined in these spaces. Note that the moduli of smoothness used in [36] and [41] are connected with differences of a function, and are inapplicable in the weighted cases. Therefore, we shall use the moduli of smoothness $\Omega_{M, \omega}^{k}(\cdot, f), k=1,2, \ldots$, defined above.

Let $W_{M}^{r}(\mathbb{T}, \omega)(r=1,2, \ldots)$ be the linear space of functions for which $f^{(r-1)}$ is absolutely continuous and $f^{(r)} \in L_{M}(\mathbb{T}, \omega)$. It becomes a Banach space with the norm

$$
\|f\|_{W_{M}^{r}(\mathbb{T}, \omega)}:=\|f\|_{L_{M}(\mathbb{T}, \omega)}+\left\|f^{(r)}\right\|_{L_{M}(\mathbb{T}, \omega)} .
$$

Our main results are the following. 
Theorem 1. Let $L_{M}(\mathbb{T}, \omega)$ be a weighted Orlicz space with Boyd indices $0<\alpha_{M} \leq \beta_{M}<1$, and let $\omega \in A_{1 / \alpha_{M}}(\mathbb{T}) \cap A_{1 / \beta_{M}}(\mathbb{T})$. Then for every $f \in W_{M}^{r}(\mathbb{T}, \omega)(r=0,1,2, \ldots)$ the inequality

$$
E_{n}(f)_{M, \omega} \leq \frac{c}{(n+1)^{r}} E_{n}\left(f^{(r)}\right)_{M, \omega}
$$

holds with a constant $c>0$ independent of $n$.

Theorem 2. Let $L_{M}(\mathbb{T}, \omega)$ be a weighted Orlicz space with Boyd indices $0<\alpha_{M} \leq \beta_{M}<1$, and let $\omega \in A_{1 / \alpha_{M}}(\mathbb{T}) \cap A_{1 / \beta_{M}}(\mathbb{T})$. Then for every $f \in L_{M}(\mathbb{T}, \omega)$ the estimate

$$
E_{n}(f)_{M, \omega} \leq c \Omega_{M, \omega}^{k}\left(\frac{1}{n+1}, f\right), \quad k=1,2, \ldots,
$$

holds with a constant $c>0$ independent of $n$.

Let $\mathbb{D}$ be the unit disk in the complex plane and $H_{1}(\mathbb{D})$ be the Hardy space of analytic functions in $\mathbb{D}$. It is known that every function $f \in H_{1}(\mathbb{D})$ admits nontangential boundary limits a.e. on the unit circle $\mathbb{T}$ and the limit function belongs to $L_{1}(\mathbb{T})[12$, p. 23].

Let $L_{M}(\mathbb{T}, \omega)$ be a weighted Orlicz space on $\mathbb{T}$ and let $H_{M}(\mathbb{D}, \omega)$ be the weighted Hardy-Orlicz class defined as

$$
H_{M}(\mathbb{D}, \omega):=\left\{f \in H_{1}(\mathbb{D}): f \in L_{M}(\mathbb{T}, \omega)\right\} .
$$

Then from Theorem 2 we obtain the following result.

Theorem 3. Let $H_{M}(\mathbb{D}, \omega)$ be a weighted Hardy-Orlicz space with Boyd indices $0<\alpha_{M} \leq \beta_{M}<1$ and let $\omega \in A_{1 / \alpha_{M}}(\mathbb{T}) \cap A_{1 / \beta_{M}}(\mathbb{T})$. If $\sum_{j=0}^{\infty} a_{j}(f) z^{j}$ is the Taylor series of $f \in H_{M}(\mathbb{D}, \omega)$ at the origin, then

$$
\left\|f(z)-\sum_{j=0}^{n} a_{j}(f) z^{j}\right\|_{L_{M}(\mathbb{T}, \omega)} \leq c \Omega_{M, \omega}^{k}\left(\frac{1}{n+1}, f\right), \quad k=1,2, \ldots,
$$

with a constant $c>0$ independent of $n$.

Our inverse results are the following.

TheOREm 4. Let $L_{M}(\mathbb{T}, \omega)$ be a weighted Orlicz space with Boyd indices $0<\alpha_{M} \leq \beta_{M}<1$, and let $\omega \in A_{1 / \alpha_{M}}(\mathbb{T}) \cap A_{1 / \beta_{M}}(\mathbb{T})$. Then for $f \in$ $L_{M}(\mathbb{T}, \omega)$ and for every natural number $n$ the estimate

$$
\Omega_{M, \omega}^{k}\left(\frac{1}{n}, f\right) \leq \frac{c}{n^{2 k}}\left\{E_{0}(f)_{M, \omega}+\sum_{m=1}^{n} m^{2 k-1} E_{m}(f)_{M, \omega}\right\},
$$

$$
k=1,2, \ldots,
$$

holds with a constant $c$ independent of $n$.

The following theorem gives a sufficient condition for $f$ to belong to $W_{M}^{2 r}(\mathbb{T}, \omega)$. 
Theorem 5. Let $L_{M}(\mathbb{T}, \omega)$ and $\omega$ be as in Theorem 4 . If $f \in L_{M}(\mathbb{T}, \omega)$ satisfies, for some $r=1,2, \ldots$,

$$
\sum_{m=1}^{\infty} m^{2 r-1} E_{m}(f)_{M, \omega}<\infty
$$

then $f \in W_{M}^{2 r}(\mathbb{T}, \omega)$.

From Theorem 4 we also have the following result.

COROLlary 1. Under the conditions of Theorem 4, if $f \in L_{M}(\mathbb{T}, \omega)$ satisfies, for some $\alpha>0$,

$$
E_{m}(f)_{M, \omega}=\mathcal{O}\left(m^{-\alpha}\right), \quad m=1,2, \ldots,
$$

then for any natural number $k$ and $\delta>0$,

$$
\Omega_{M, \omega}^{k}(\delta, f)= \begin{cases}\mathcal{O}\left(\delta^{\alpha}\right), & k>\alpha / 2, \\ \mathcal{O}\left(\delta^{\alpha} \log (1 / \delta)\right), & k=\alpha / 2, \\ \mathcal{O}\left(\delta^{2 k}\right), & k<\alpha / 2 .\end{cases}
$$

Hence if we define the generalized Lipschitz class Lip* $\alpha(M, \omega)$ for $\alpha>0$ and $k:=[\alpha / 2]+1$ as

$$
\operatorname{Lip}^{*} \alpha(M, \omega):=\left\{f \in L_{M}(\mathbb{T}, \omega): \Omega_{M, \omega}^{k}(\delta, f) \leq c \delta^{\alpha}, \delta>0\right\},
$$

then from Corollary 1 we obtain the following

COROLlary 2. Under the conditions of Theorem 4, if $f \in L_{M}(\mathbb{T}, \omega)$ satisfies, for some $\alpha>0$,

$$
E_{m}(f)_{M, \omega}=\mathcal{O}\left(m^{-\alpha}\right), \quad m=1,2, \ldots,
$$

then $f \in \operatorname{Lip}^{*} \alpha(M, \omega)$.

Combining this with Theorem 2 we get the following constructive description of the classes Lip* $\alpha(M, \omega)$.

Theorem 6. Let $L_{M}(\mathbb{T}, \omega)$ be a weighted Orlicz space with Boyd indices $0<\alpha_{M} \leq \beta_{M}<1$, and let $\omega \in A_{1 / \alpha_{M}}(\mathbb{T}) \cap A_{1 / \beta_{M}}(\mathbb{T})$. Then for $\alpha>0$ the following assertions are equivalent:

(i) $f \in \operatorname{Lip}^{*} \alpha(M, \omega)$;

(ii) $E_{m}(f)_{M, \omega}=\mathcal{O}\left(m^{-\alpha}\right)$.

REMARK 1. The assumptions

$$
0<\alpha_{M} \leq \beta_{M}<1 \quad \text { and } \omega \in A_{1 / \alpha_{M}}(\mathbb{T}) \cap A_{1 / \beta_{M}}(\mathbb{T})
$$

are important in our investigations. In particular, they guarantee the existence and boundedness of the operator $\sigma_{h}$ (see Lemma 1) and also the well-definedness of the $k$-modulus of smoothness $\Omega_{M, \omega}^{k}(\cdot, f)(k=1,2, \ldots)$, in terms of which we estimate the error of approximation in weighted Orlicz spaces $L_{M}(\mathbb{T}, \omega)$. 
We use $c, c_{1}, c_{2}, \ldots$ to denote constants (which may, in general, differ in different relations) depending only on numbers that are not important for the questions of interest.

2. Auxiliary results. The following interpolation theorem was proved in [4] (see also [2, p. 153]).

TheOREM 7. Let $1<q<p<\infty$. If a linear operator is bounded in the Lebesgue spaces $L_{p}(\mathbb{T})$ and $L_{q}(\mathbb{T})$, then it is bounded in every Orlicz space $L_{M}(\mathbb{T})$ whose Boyd indices satisfy $1 / p<\alpha_{M} \leq \beta_{M}<1 / q$.

Using this theorem we obtain the following result about the boundedness of the linear operator $\sigma_{h}$ in weighted Orlicz spaces.

Lemma 1. Let $L_{M}(\mathbb{T}, \omega)$ have Boyd indices $0<\alpha_{M} \leq \beta_{M}<1$. If $\omega \in$ $A_{1 / \alpha_{M}}(\mathbb{T}) \cap A_{1 / \beta_{M}}(\mathbb{T})$, then the operator $\sigma_{h}$ is bounded in $L_{M}(\mathbb{T}, \omega)$.

Proof. Since $0<\alpha_{M} \leq \beta_{M}<1$, by Theorem 2.31 from [3, p. 58] we can find numbers $q$ and $p$ such that

$$
1<q<1 / \beta_{M} \leq 1 / \alpha_{M}<p<\infty
$$

and $\omega$ belongs to $A_{p}(\mathbb{T})$ and $A_{q}(\mathbb{T})$. Then it follows from the continuity of the maximal operator in weighted Lebesgue spaces [35] (see also [13, p. 110]) that $\sigma_{h}$ is bounded in $L_{p}(\mathbb{T}, \omega)$ and $L_{q}(\mathbb{T}, \omega)$. In that case the operator $A_{h}:=\omega \sigma_{h} \omega^{-1} I$ is bounded in $L_{p}(\mathbb{T})$ and $L_{q}(\mathbb{T})$. Hence by Theorem $7, A_{h}$ is bounded in $L_{M}(\mathbb{T})$. This implies the boundedness of $\sigma_{h}$ in $L_{M}(\mathbb{T}, \omega)$.

Corollary 3. Let $L_{M}(\mathbb{T}, \omega)$ have Boyd indices $0<\alpha_{M} \leq \beta_{M}<1$. If $\omega \in A_{1 / \alpha_{M}}(\mathbb{T}) \cap A_{1 / \beta_{M}}(\mathbb{T})$, then

$$
\lim _{h \rightarrow 0}\left\|f-\sigma_{h} f\right\|_{L_{M}(\mathbb{T}, \omega)}=0
$$

for $f \in L_{M}(\mathbb{T}, \omega)$ and hence

$$
\lim _{\delta \rightarrow 0} \Omega_{M, \omega}^{k}(\delta, f)=0, \quad k=1,2, \ldots
$$

Moreover

$$
\Omega_{M, \omega}^{k}(\delta, f) \leq c\|f\|_{L_{M}(\mathbb{T}, \omega)}
$$

with some constant $c$ independent of $f$.

Let $S_{n}(\cdot, f)(n=1,2, \ldots)$ be the $n$th partial sum of the Fourier series of $f \in L_{1}(\mathbb{T})$, i.e.

$$
S_{n}(x, f)=\frac{a_{0}}{2}+\sum_{k=1}^{n}\left(a_{k} \cos k x+b_{k} \sin k x\right) .
$$

Then [1, Vol. 1, pp. 95-96]

$$
S_{n}(x, f)=\frac{1}{\pi} \int_{\mathbb{T}} f(t) D_{n}(x-t) d t
$$


with the Dirichlet kernel

$$
D_{n}(t):=\frac{1}{2}+\sum_{k=1}^{n} \cos k t
$$

of order $n$. Consider the sequence $\left\{K_{n}(\cdot, f)\right\}$ of the arithmetic means of the partial sums of the Fourier series of $f$, that is,

$$
K_{n}(x, f):=\frac{S_{0}(x, f)+S_{1}(x, f)+\cdots+S_{n}(x, f)}{n+1}, \quad n=0,1,2, \ldots,
$$

with $K_{0}(x, f)=S_{0}(x, f):=a_{0} / 2$.

It is known [1, Vol. 1, p. 133] that

$$
K_{n}(x, f)=\frac{1}{\pi} \int_{\mathbb{T}} f(t) F_{n}(x-t) d t,
$$

where

$$
F_{n}(t):=\frac{1}{n+1} \sum_{k=0}^{n} D_{k}(t)
$$

is the Fejér kernel of order $n$ (for more information see [1, Vol. 1, pp. 133137]).

Since the linear operator $K_{n}$ is bounded in $L_{p}(\mathbb{T}, \omega)$ and $L_{q}(\mathbb{T}, \omega)$ (see [38], [35], also [13, p. 109]), the proof of the following result is similar to that of Lemma 1.

Lemma 2. Let $L_{M}(\mathbb{T}, \omega)$ have Boyd indices $0<\alpha_{M} \leq \beta_{M}<1$. If $\omega \in$ $A_{1 / \alpha_{M}}(\mathbb{T}) \cap A_{1 / \beta_{M}}(\mathbb{T})$, then the operator $K_{n}$ is bounded in $L_{M}(\mathbb{T}, \omega)$, i.e.

$$
\left\|K_{n}(\cdot, f)\right\|_{L_{M}(\mathbb{T}, \omega)} \leq c\|f\|_{L_{M}(\mathbb{T}, \omega)}, \quad f \in L_{M}(\mathbb{T}, \omega),
$$

with a constant $c$ independent of $n$.

Now we can state and prove the Bernstein inequality for weighted Orlicz spaces.

Lemma 3. Let $L_{M}(\mathbb{T}, \omega)$ have Boyd indices $0<\alpha_{M} \leq \beta_{M}<1$. If $\omega \in$ $A_{1 / \alpha_{M}}(\mathbb{T}) \cap A_{1 / \beta_{M}}(\mathbb{T})$, then for each trigonometric polynomial $T_{n}$ of degree $n$ the inequality

$$
\left\|T_{n}^{\prime}\right\|_{L_{M}(\mathbb{T}, \omega)} \leq c n\left\|T_{n}\right\|_{L_{M}(\mathbb{T}, \omega)}
$$

holds with a constant $c$ independent of $n$.

Proof. We use Zygmund's method (see [1, Vol. 2, pp. 458-460]). Since

$$
T_{n}(x)=S_{n}\left(x, T_{n}\right)=\frac{1}{\pi} \int_{\mathbb{T}} T_{n}(u) D_{n}(u-x) d u,
$$


by differentiation we get

$$
\begin{aligned}
T_{n}^{\prime}(x) & =-\frac{1}{\pi} \int_{\mathbb{T}} T_{n}(u) D_{n}^{\prime}(u-x) d u=-\frac{1}{\pi} \int_{\mathbb{T}} T_{n}(u+x) D_{n}^{\prime}(u) d u \\
& =\frac{1}{\pi} \int_{\mathbb{T}} T_{n}(u+x)\left(\sum_{k=1}^{n} k \sin k u\right) d u .
\end{aligned}
$$

Noting that

$$
\int_{\mathbb{T}} T_{n}(u+x) \sum_{k=1}^{n-1} k \sin (2 n-k) u d u=0
$$

we have

$$
\begin{aligned}
T_{n}^{\prime}(x) & =\frac{1}{\pi} \int_{\mathbb{T}} T_{n}(u+x)\left\{\sum_{k=1}^{n} k \sin k u+\sum_{k=1}^{n-1} k \sin (2 n-k) u\right\} d u \\
& =\frac{1}{\pi} \int_{\mathbb{T}} T_{n}(u+x) 2 n \sin n u\left\{\frac{1}{2}+\sum_{k=1}^{n-1} \frac{n-k}{n} \cos k u\right\} d u \\
& =2 n \frac{1}{\pi} \int_{\mathbb{T}} T_{n}(u+x) \sin n u F_{n-1}(u) d u .
\end{aligned}
$$

Since $F_{n-1}$ is nonnegative, this implies

$$
\begin{aligned}
\left|T_{n}^{\prime}(x)\right| & \leq 2 n \frac{1}{\pi} \int_{\mathbb{T}}\left|T_{n}(u+x)\right| F_{n-1}(u) d u \\
& =2 n \frac{1}{\pi} \int_{\mathbb{T}}\left|T_{n}(u)\right| F_{n-1}(u-x) d u=2 n K_{n-1}\left(x,\left|T_{n}\right|\right) .
\end{aligned}
$$

The last inequality and (13) yield (14).

REMARK 2. The Bernstein inequality in $L_{p}(\mathbb{T}, \omega)$ was proved in [29] for more general doubling weights. Generalizing it to more general weights is not our goal in this work and the above inequality is sufficient for our considerations.

Taking the boundedness of the linear operators $f \mapsto S_{n}(\cdot, f)$ and $f \mapsto \widetilde{f}$ in $L_{p}(\mathbb{T}, \omega)$ into account $[17,18]$ and using the method of proof of Lemma 1 , one can show that

$$
\left\|S_{n}(\cdot, f)\right\|_{L_{M}(\mathbb{T}, \omega)} \leq c\|f\|_{L_{M}(\mathbb{T}, \omega)}, \quad\|\widetilde{f}\|_{L_{M}(\mathbb{T}, \omega)} \leq c\|f\|_{L_{M}(\mathbb{T}, \omega)},
$$

and as a corollary we obtain

$$
\left\|f-S_{n}(\cdot, f)\right\|_{L_{M}(\mathbb{T}, \omega)} \leq c E_{n}(f)_{M, \omega}, \quad E_{n}(\widetilde{f})_{M, \omega} \leq c E_{n}(f)_{M, \omega},
$$

where $\tilde{f}$ is the conjugate function of $f \in L_{M}(\mathbb{T}, \omega)$. 
Lemma 4. Let $L_{M}(\mathbb{T}, \omega)$ have Boyd indices $0<\alpha_{M} \leq \beta_{M}<1$. If $\omega \in$ $A_{1 / \alpha_{M}}(\mathbb{T}) \cap A_{1 / \beta_{M}}(\mathbb{T})$, then the class of trigonometric polynomials is dense in $L_{M}(\mathbb{T}, \omega)$.

Proof. Let $L_{M}(\mathbb{T})$ be an Orlicz space with $0<\alpha_{M} \leq \beta_{M}<1$. Then $L_{M}(\mathbb{T})$ is reflexive. On the other hand, from the method of proof of Theorem 4.5 in [24] and Lemma 4.2 in [22], it can be deduced that the conditions $\omega \in$ $A_{1 / \alpha_{M}}(\mathbb{T})$ and $\omega \in A_{1 / \beta_{M}}(\mathbb{T})$ imply that $\omega \in L_{M}(\mathbb{T})$ and $1 / \omega \in L_{N}(\mathbb{T})$. Then the space $L_{M}(\mathbb{T}, \omega)$ is also reflexive [25, Corollary 2.8] and by Lemmas 1.2 and 1.3 in [23] the class $C(\mathbb{T})$ of continuous functions is dense in $L_{M}(\mathbb{T}, \omega)$.

Let $f \in L_{M}(\mathbb{T}, \omega)$ and $\varepsilon>0$. Since $C(\mathbb{T})$ is dense in $L_{M}(\mathbb{T}, \omega)$, there is a continuous function $f_{0}$ such that

$$
\left\|f-f_{0}\right\|_{L_{M}(\mathbb{T}, \omega)}<\varepsilon
$$

By the Weierstrass theorem, there exists a trigonometric polynomial $T_{0}$ such that

$$
\left|f_{0}(x)-T_{0}(x)\right|<\varepsilon, \quad x \in \mathbb{T} .
$$

Using this and formulas (3), (5) and the Hölder inequality we get

$$
\begin{aligned}
\left\|f_{0}-T_{0}\right\|_{L_{M}(\mathbb{T}, \omega)} & =\left\|\left(f_{0}-T_{0}\right) \omega\right\|_{L_{M}(\mathbb{T})} \\
& =\sup \left\{\int_{\mathbb{T}}\left|f_{0}(x)-T_{0}(x)\right| \omega(x)|g(x)| d x:\|g\|_{L_{N}(\mathbb{T})}^{*} \leq 1\right\} \\
& \leq \varepsilon \sup \left\{\int_{\mathbb{T}} \omega(x)|g(x)| d x:\|g\|_{L_{N}(\mathbb{T})}^{*} \leq 1\right\} \\
& \leq \varepsilon \sup \left\{\|\omega\|_{L_{M}(\mathbb{T})}\|g\|_{L_{N}(\mathbb{T})}^{*}:\|g\|_{L_{N}(\mathbb{T})}^{*} \leq 1\right\} \leq \varepsilon\|\omega\|_{L_{M}(\mathbb{T})},
\end{aligned}
$$

which by (17) yields

$$
\left\|f-T_{0}\right\|_{L_{M}(\mathbb{T}, \omega)} \leq\left\|f-f_{0}\right\|_{L_{M}(\mathbb{T}, \omega)}+\left\|f_{0}-T_{0}\right\|_{L_{M}(\mathbb{T}, \omega)}<\left(1+\|\omega\|_{L_{M}(\mathbb{T})}\right) \varepsilon,
$$

and the assertion is proved.

Corollary 4. Let $L_{M}(\mathbb{T}, \omega)$ have Boyd indices $0<\alpha_{M} \leq \beta_{M}<1$. If $\omega \in A_{1 / \alpha_{M}}(\mathbb{T}) \cap A_{1 / \beta_{M}}(\mathbb{T})$, then the Fourier series of $f \in L_{M}(\mathbb{T}, \omega)$ converges to $f$ in the norm of $L_{M}(\mathbb{T}, \omega)$.

Proof. By Lemma 4 we have $E_{n}(f)_{M, \omega} \rightarrow 0(n \rightarrow \infty)$ and then the assertion follows from (16).

Lemma 5. If $\omega \in A_{1 / \alpha_{M}}(\mathbb{T}) \cap A_{1 / \beta_{M}}(\mathbb{T})$ and $f \in W_{M}^{2}(\mathbb{T}, \omega)$, then

$$
\Omega_{M, \omega}^{k}(\delta, f) \leq c \delta^{2} \Omega_{M, \omega}^{k-1}\left(\delta, f^{\prime \prime}\right), \quad k=1,2, \ldots,
$$

with some constant $c$ independent of $\delta$.

Proof. Consider the function

$$
g(x):=\prod_{i=2}^{k}\left(I-\sigma_{h_{i}}\right) f(x) .
$$


Then $g \in W_{M}^{2}(\mathbb{T}, \omega)$ and

$$
\left(I-\sigma_{h_{1}}\right) g(x)=\left(I-\sigma_{h_{1}}\right)\left(\prod_{i=2}^{k}\left(I-\sigma_{h_{i}}\right) f(x)\right)=\prod_{i=1}^{k}\left(I-\sigma_{h_{i}}\right) f(x) .
$$

Hence

$$
\begin{aligned}
\prod_{i=1}^{k}\left(I-\sigma_{h_{i}}\right) f(x) & =g(x)-\sigma_{h_{1}}(x)=g(x)-\frac{1}{2 h_{1}} \int_{-h_{1}}^{h_{1}} g(x+t) d t \\
& =\frac{1}{2 h_{1}} \int_{-h_{1}}^{h_{1}}[g(x)-g(x+t)] d t \\
& =-\frac{1}{4 h_{1}} \int_{-h_{1}}^{h_{1}}[g(x+t)-2 g(x)+g(x-t)] d t \\
& =-\frac{1}{8 h_{1}} \int_{0}^{h_{1}} \int_{0}^{t} \int_{-u}^{u} g^{\prime \prime}(x+s) d s d u d t .
\end{aligned}
$$

Now, according to (1), (5) and Fubini's theorem and moving the supremum under the integral sign we have

$$
\begin{aligned}
\left\|\prod_{i=1}^{k}\left(I-\sigma_{h_{i}}\right) f\right\|_{L_{M}(\mathbb{T}, \omega)} & =\frac{1}{8 h_{1}}\left\|\int_{0}^{h_{1}} \int_{0}^{t} \int_{-u}^{u} g^{\prime \prime}(\cdot+s) d s d u d t\right\|_{L_{M}(\mathbb{T}, \omega)} \\
& =\frac{1}{8 h_{1}} \sup \int\left|\int_{\mathbb{T}} \int_{0} \int_{0}^{t} \int_{-u}^{u} g^{\prime \prime}(x+s) d s d u d t\right| \omega(x)|l(x)| d x \\
& \leq \frac{1}{8 h_{1}} \sup \int\left[\int_{\mathbb{T}}^{h_{1}} \int_{0}^{t}\left|\int_{-u}^{u} g^{\prime \prime}(x+s) d s\right| d u d t\right] \omega(x)|l(x)| d x \\
& =\frac{1}{8 h_{1}} \sup \int_{0}^{h_{1}} \int_{0}^{t}\left[\int_{\mathbb{T}}^{u}\left|\int_{-u}^{u} g^{\prime \prime}(x+s) d s\right| \omega(x)|l(x)| d x\right] d u d t \\
& \leq \frac{1}{8 h_{1}} \int_{0}^{h_{1}} \int_{0}^{t}\left[\sup \int\left|\int_{\mathbb{T}}^{u} g^{\prime \prime}(x+s) d s\right| \omega(x)|l(x)| d x\right] d u d t \\
& =\frac{1}{8 h_{1}} \int_{0}^{h_{1}} \int_{0}^{t}\left\|\int_{-u}^{u} g^{\prime \prime}(\cdot+s) d s\right\|_{L_{M}(\mathbb{T}, \omega)} d u d t \\
& =\frac{1}{8 h_{1}} \int_{0}^{h_{1}} \int_{0}^{t} 2 u\left\|\frac{1}{2 u} \int_{-u}^{u} g^{\prime \prime}(\cdot+s) d s\right\|_{L_{M}(\mathbb{T}, \omega)} d u d t
\end{aligned}
$$

where the suprema are taken over all $l \in \widetilde{L}_{N}(\mathbb{T})$ with $\|l\|_{L_{N}(\mathbb{T})}^{*} \leq 1$. Taking 
into account the boundedness of $\sigma_{u}$ we see that

$$
\begin{aligned}
\left\|\prod_{i=1}^{k}\left(I-\sigma_{h_{i}}\right) f\right\|_{L_{M}(\mathbb{T}, \omega)} & \leq \frac{1}{8 h_{1}} \int_{0}^{h_{1}} \int_{0}^{t} 2 u\left\|\sigma_{u} g^{\prime \prime}\right\|_{L_{M}(\mathbb{T}, \omega)} d u d t \\
& \leq c \frac{1}{8 h_{1}} \int_{0}^{h_{1} t} \int_{0}^{t} 2 u\left\|g^{\prime \prime}\right\|_{L_{M}(\mathbb{T}, \omega)} d u d t=c h_{1}^{2}\left\|g^{\prime \prime}\right\|_{L_{M}(\mathbb{T}, \omega)} .
\end{aligned}
$$

On the other hand, $g^{\prime \prime}=\prod_{i=2}^{k}\left(I-\sigma_{h_{i}}\right) f^{\prime \prime}$ by the definitions of $g$ and $\sigma_{h_{i}}$. Then from the last inequality we conclude that

$$
\begin{aligned}
\Omega_{M, \omega}^{k}(\delta, f) & =\sup _{\substack{0<h_{i} \leq \delta \\
1 \leq i \leq k}}\left\|\prod_{i=1}^{k}\left(I-\sigma_{h_{i}}\right) f\right\|_{L_{M}(\mathbb{T}, \omega)} \leq \sup _{\substack{0<h_{i} \leq \delta \\
1 \leq i \leq k}} c h_{1}^{2}\left\|g^{\prime \prime}\right\|_{L_{M}(\mathbb{T}, \omega)} \\
& =c \delta_{1}^{2} \sup _{\substack{0<h_{i} \leq \delta \\
2 \leq i \leq k}}\left\|\prod_{i=2}^{k}\left(I-\sigma_{h_{i}}\right) f^{\prime \prime}\right\|_{L_{M}(\mathbb{T}, \omega)}=c \delta^{2} \Omega_{M, \omega}^{k-1}\left(\delta, f^{\prime \prime}\right) .
\end{aligned}
$$

Corollary 5. If $f \in W_{M}^{2 k}(\mathbb{T}, \omega)$, then

$$
\Omega_{M, \omega}^{k}(\delta, f) \leq c \delta^{2 k}\left\|f^{(2 k)}\right\|_{L_{M}(\mathbb{T}, \omega)}
$$

with some constant $c$ independent of $\delta$.

For $f \in L_{M}(\mathbb{T}, \omega)$ and $\delta>0$, the $K$-functional is defined as

$$
\begin{aligned}
K\left(\delta, f ; L_{M}\right. & \left.(\mathbb{T}, \omega), W_{M}^{r}(\mathbb{T}, \omega)\right) \\
& :=\inf \left\{\|f-\psi\|_{L_{M}(\mathbb{T}, \omega)}+\delta\left\|\psi^{(r)}\right\|_{L_{M}(\mathbb{T}, \omega)}: \psi \in W_{M}^{r}(\mathbb{T}, \omega)\right\} .
\end{aligned}
$$

Theorem 8. Let $L_{M}(\mathbb{T}, \omega)$ be a weighted Orlicz space with Boyd indices $0<\alpha_{M} \leq \beta_{M}<1$. If $\omega \in A_{1 / \alpha_{M}}(\mathbb{T}) \cap A_{1 / \beta_{M}}(\mathbb{T})$, then for $f \in L_{M}(\mathbb{T}, \omega)$ and $k=1,2, \ldots$ the equivalence

$$
K\left(\delta^{2 k}, f ; L_{M}(\mathbb{T}, \omega), W_{M}^{2 k}(\mathbb{T}, \omega)\right) \sim \Omega_{M, \omega}^{k}(\delta, f)
$$

holds, where the implied constants are independent of $\delta$.

Proof. Let $\psi$ be an arbitrary function in $W_{M}^{2 k}(\mathbb{T}, \omega)$. By (6) and Corollaries 3 and 5 we obtain

$$
\begin{aligned}
\Omega_{M, \omega}^{k}(\delta, f) & =\Omega_{M, \omega}^{k}(\delta, f-\psi+\psi) \leq \Omega_{M, \omega}^{k}(\delta, f-\psi)+\Omega_{M, \omega}^{k}(\delta, \psi) \\
& \leq c_{1}\|f-\psi\|_{L_{M}(\mathbb{T}, \omega)}+c_{2} \delta^{2 k}\left\|\psi^{(2 k)}\right\|_{L_{M}(\mathbb{T}, \omega)} .
\end{aligned}
$$

If we take the infimum over all $\psi \in W_{M}^{2 k}(\mathbb{T}, \omega)$, then by definition of the $K$-functional we get

$$
\Omega_{M, \omega}^{k}(\delta, f) \leq c K\left(\delta^{2 k}, f ; L_{M}(\mathbb{T}, \omega), W_{M}^{2 k}(\mathbb{T}, \omega)\right) .
$$


For the proof of the reverse estimate consider an operator $L_{\delta}$ on $L_{M}(\mathbb{T}, \omega)$ given by

$$
\left(L_{\delta} f\right)(x):=\frac{3}{\delta^{3}} \int_{0}^{\delta} \int_{0}^{u} \int_{-t}^{t} f(x+s) d s d t d u, \quad x \in \mathbb{T} .
$$

Then

$$
\frac{d^{2}}{d x^{2}}\left(L_{\delta} f\right)=\frac{c}{\delta^{2}}\left(I-\sigma_{\delta}\right) f
$$

and hence

$$
\frac{d^{2 k}}{d x^{2 k}} L_{\delta}^{k}=\frac{c}{\delta^{2 k}}\left(I-\sigma_{\delta}\right)^{k}, \quad k=1,2, \ldots
$$

The operator $L_{\delta}$ is bounded in $L_{M}(\mathbb{T}, \omega)$. Indeed, applying Minkowski's inequality and the boundedness of $\sigma_{t}$ in $L_{M}(\mathbb{T}, \omega)$ we get

$$
\begin{aligned}
\left\|L_{\delta} f\right\|_{L_{M}(\mathbb{T}, \omega)} & \leq \frac{3}{\delta^{3}} \int_{0}^{\delta} \int_{0}^{u}\left\|\int_{-t}^{t} f(\cdot+s) d s\right\|_{L_{M}(\mathbb{T}, \omega)} d t d u \\
& =\frac{3}{\delta^{3}} \int_{0}^{\delta} \int_{0}^{u} 2 t\left\|\sigma_{t} f\right\|_{L_{M}(\mathbb{T}, \omega)} d t d u \\
& \leq c \frac{3}{\delta^{3}}\|f\|_{L_{M}(\mathbb{T}, \omega)} \iint_{0}^{\delta} 2 t d t d u=c\|f\|_{L_{M}(\mathbb{T}, \omega)} .
\end{aligned}
$$

Define another operator $A_{\delta}^{k}$ by

$$
A_{\delta}^{k}:=I-\left(I-L_{\delta}^{k}\right)^{k} .
$$

Then $A_{\delta}^{k} f \in W_{M}^{2 k}(\mathbb{T}, \omega)$ for $f \in L_{M}(\mathbb{T}, \omega)$ and furthermore, by (19),

$$
\begin{aligned}
\left\|\frac{d^{2 k}}{d x^{2 k}} A_{\delta}^{k} f\right\|_{L_{M}(\mathbb{T}, \omega)} & \leq c\left\|\frac{d^{2 k}}{d x^{2 k}} L_{\delta}^{k} f\right\|_{L_{M}(T, \omega)} \\
& =\frac{c}{\delta^{2 k}}\left\|\left(I-\sigma_{\delta}\right)^{k} f\right\|_{L_{M}(T, \omega)}
\end{aligned}
$$

This inequality and the definition of $\Omega_{M, \omega}^{k}(\delta, f)$ yield

$$
\delta^{2 k}\left\|\frac{d^{2 k}}{d x^{2 k}} A_{\delta}^{k} f\right\|_{L_{M}(T, \omega)} \leq c \Omega_{M, \omega}^{k}(\delta, f) .
$$

Since

$$
I-L_{\delta}^{k}=\left(I-L_{\delta}\right) \sum_{j=0}^{k-1} L_{\delta}^{j}
$$


and $L_{\delta}$ is bounded in $L_{M}(\mathbb{T}, \omega)$, we have

$$
\begin{aligned}
& \left\|\left(I-L_{\delta}^{k}\right) g\right\|_{L_{M}(\mathbb{T}, \omega)}=\left\|\left(\sum_{j=0}^{k-1} L_{\delta}^{j}\right)\left(I-L_{\delta}\right) g\right\|_{L_{M}(\mathbb{T}, \omega)} \\
& \leq c\left\|\left(I-L_{\delta}\right) g\right\|_{L_{M}(\mathbb{T}, \omega)} \\
& =c\left\|\frac{3}{\delta^{3}} \int_{0}^{\delta} \int_{0}^{u} \int_{-t}^{t}[g-g(\cdot+s)] d s d t d u\right\|_{L_{M}(\mathbb{T}, \omega)} \\
& \leq \frac{3 c}{\delta^{3}} \int_{0}^{\delta} \int_{0}^{u} 2 t\left\|\frac{1}{2 t} \int_{-t}^{t}[g-g(\cdot+s)] d s\right\|_{L_{M}(\mathbb{T}, \omega)} d t d u \\
& =\frac{3 c}{\delta^{3}} \int_{0}^{\delta} \int_{0}^{u} 2 t\left\|\left(I-\sigma_{t}\right) g\right\|_{L_{M}(\mathbb{T}, \omega)} d t d u \\
& \leq \frac{3 c}{\delta^{3}} \sup _{0<t \leq \delta}\left\|\left(I-\sigma_{t}\right) g\right\|_{L_{M}(\mathbb{T}, \omega)} \int_{0}^{\delta} \int_{0}^{u} 2 t d t d u \\
& =c \sup _{0<t \leq \delta}\left\|\left(I-\sigma_{t}\right) g\right\|_{L_{M}(\mathbb{T}, \omega)}
\end{aligned}
$$

for every $g \in L_{M}(\mathbb{T}, \omega)$. Applying this inequality $k$ times in

$\left\|f-A_{\delta}^{k} f\right\|_{L_{M}(\mathbb{T}, \omega)}=\left\|\left(I-L_{\delta}^{k}\right)^{k} f\right\|_{L_{M}(\mathbb{T}, \omega)}=\left\|\left(I-L_{\delta}^{k}\right)\left(I-L_{\delta}^{k}\right)^{k-1} f\right\|_{L_{M}(\mathbb{T}, \omega)}$

we obtain

$$
\begin{aligned}
\left\|f-A_{\delta}^{k} f\right\|_{L_{M}(\mathbb{T}, \omega)} & \leq c_{1} \sup _{0<t_{1} \leq \delta}\left\|\left(I-\sigma_{t_{1}}\right)\left(I-L_{\delta}^{k}\right)^{k-1} f\right\|_{L_{M}(\mathbb{T}, \omega)} \\
& \leq c_{2} \sup _{0<t_{1} \leq \delta} \sup _{0<t_{2} \leq \delta}\left\|\left(I-\sigma_{t_{1}}\right)\left(I-\sigma_{t_{2}}\right)\left(I-L_{\delta}^{k}\right)^{k-2} f\right\|_{L_{M}(\mathbb{T}, \omega)} \\
& \leq \cdots \leq c \sup _{\substack{0<t_{j} \leq \delta \\
1 \leq j \leq k}}\left\|\prod_{j=1}^{k}\left(I-\sigma_{t_{j}}\right) f\right\|_{L_{M}(\mathbb{T}, \omega)}=c \Omega_{M, \omega}^{k}(\delta, f) .
\end{aligned}
$$

Since $A_{\delta}^{k} f \in W_{M}^{2 k}(\mathbb{T}, \omega)$, from the last inequality, (20) and the definition of the $K$-functional, we conclude that

$$
\begin{aligned}
& K\left(\delta^{2 k}, f ; L_{M}(\mathbb{T}, \omega), W_{M}^{2 k}(\mathbb{T}, \omega)\right) \\
& \quad \leq\left\|f-A_{\delta}^{k} f\right\|_{L_{M}(\mathbb{T}, \omega)}+\delta^{2 k}\left\|\frac{d^{2 k}}{d x^{2 k}} A_{\delta}^{k} f\right\|_{L_{M}(\mathbb{T}, \omega)} \leq c \Omega_{M, \omega}^{k}(\delta, f),
\end{aligned}
$$

which gives the reverse estimate and completes the proof.

\section{Proofs of the theorems}

Proof of Theorem 1. Let $\sum_{k=0}^{\infty}\left(a_{k} \cos k x+b_{k} \sin k x\right)$ be the Fourier series of $f$ and $S_{n}(x, f)$ be its $n$th partial sum, i.e., 


$$
S_{n}(x, f)=\sum_{k=0}^{n}\left(a_{k} \cos k x+b_{k} \sin k x\right) .
$$

It is known that the conjugate function $\widetilde{f}$ has the Fourier expansion

$$
\sum_{k=1}^{\infty}\left(a_{k} \sin k x-b_{k} \cos k x\right) \text {. }
$$

If we set

$$
A_{k}(x, f):=a_{k} \cos k x+b_{k} \sin k x,
$$

then by Corollary 4 we have

$$
f(x)=\sum_{k=0}^{\infty} A_{k}(x, f)
$$

in the norm of $L_{M}(\mathbb{T}, \omega)$. Since for $k=1,2, \ldots$,

$$
\begin{aligned}
A_{k}(x, f)= & a_{k} \cos k x+b_{k} \sin k x \\
= & a_{k} \cos k\left(x+\frac{r \pi}{2 k}-\frac{r \pi}{2 k}\right)+b_{k} \sin k\left(x+\frac{r \pi}{2 k}-\frac{r \pi}{2 k}\right) \\
= & a_{k} \cos \left(k x+\frac{r \pi}{2}-\frac{r \pi}{2}\right)+b_{k} \sin \left(k x+\frac{r \pi}{2}-\frac{r \pi}{2}\right) \\
= & a_{k}\left[\cos \left(k x+\frac{r \pi}{2}\right) \cos \frac{r \pi}{2}+\sin \left(k x+\frac{r \pi}{2}\right) \sin \frac{r \pi}{2}\right] \\
& +b_{k}\left[\sin \left(k x+\frac{r \pi}{2}\right) \cos \frac{r \pi}{2}-\cos \left(k x+\frac{r \pi}{2}\right) \sin \frac{r \pi}{2}\right] \\
= & \cos \frac{r \pi}{2}\left[a_{k} \cos k\left(x+\frac{r \pi}{2 k}\right)+b_{k} \sin k\left(x+\frac{r \pi}{2 k}\right)\right] \\
& +\sin \frac{r \pi}{2}\left[a_{k} \sin k\left(x+\frac{r \pi}{2 k}\right)-b_{k} \cos k\left(x+\frac{r \pi}{2 k}\right)\right] \\
= & A_{k}\left(x+\frac{r \pi}{2 k}, f\right) \cos \frac{r \pi}{2}+A_{k}\left(x+\frac{r \pi}{2 k}, \tilde{f}\right) \sin \frac{r \pi}{2}
\end{aligned}
$$

and

$$
A_{k}\left(x, f^{(r)}\right)=k^{r} A_{k}\left(x+\frac{r \pi}{2 k}, f\right)
$$

we get

$$
\begin{aligned}
\sum_{k=0}^{\infty} A_{k}(x, f)= & A_{0}(x, f)+\cos \frac{r \pi}{2} \sum_{k=1}^{\infty} A_{k}\left(x+\frac{r \pi}{2 k}, f\right) \\
& +\sin \frac{r \pi}{2} \sum_{k=1}^{\infty} A_{k}\left(x+\frac{r \pi}{2 k}, \tilde{f}\right)
\end{aligned}
$$




$$
\begin{aligned}
= & A_{0}(x, f)+\cos \frac{r \pi}{2} \sum_{k=1}^{\infty} \frac{1}{r^{k}} r^{k} A_{k}\left(x+\frac{r \pi}{2 k}, f\right) \\
& +\sin \frac{r \pi}{2} \sum_{k=1}^{\infty} \frac{1}{r^{k}} r^{k} A_{k}\left(x+\frac{r \pi}{2 k}, \tilde{f}\right) \\
= & A_{0}(x, f)+\cos \frac{r \pi}{2} \sum_{k=1}^{\infty} \frac{1}{r^{k}} A_{k}\left(x, f^{(r)}\right)+\sin \frac{r \pi}{2} \sum_{k=1}^{\infty} \frac{1}{r^{k}} A_{k}\left(x, \widetilde{f}^{(r)}\right)
\end{aligned}
$$

Then

$$
\begin{aligned}
f(x)-S_{n}(x, f) & =\sum_{k=n+1}^{\infty} A_{k}(x, f) \\
& =\cos \frac{r \pi}{2} \sum_{k=n+1}^{\infty} \frac{1}{r^{k}} A_{k}\left(x, f^{(r)}\right)+\sin \frac{r \pi}{2} \sum_{k=n+1}^{\infty} \frac{1}{r^{k}} A_{k}\left(x, \tilde{f}^{(r)}\right) .
\end{aligned}
$$

Taking into account that

$$
\begin{aligned}
\sum_{k=n+1}^{\infty} \frac{1}{k^{r}} A_{k}\left(\theta, f^{(r)}\right)= & \sum_{k=n+1}^{\infty} \frac{1}{k^{r}}\left[S_{k}\left(\theta, f^{(r)}\right)-S_{k-1}\left(\theta, f^{(r)}\right)\right] \\
= & \sum_{k=n+1}^{\infty} \frac{1}{k^{r}}\left\{\left[S_{k}\left(\theta, f^{(r)}\right)-f^{(r)}(\theta)\right]-\left[S_{k-1}\left(\theta, f^{(r)}\right)-f^{(r)}(\theta)\right]\right\} \\
= & \sum_{k=n+1}^{\infty}\left(\frac{1}{k^{r}}-\frac{1}{(k+1)^{r}}\right)\left[S_{k}\left(\theta, f^{(r)}\right)-f^{(r)}(\theta)\right] \\
& -\frac{1}{(n+1)^{r}}\left[S_{n}\left(\theta, f^{(r)}\right)-f^{(r)}(\theta)\right],
\end{aligned}
$$

and

$$
\begin{aligned}
\sum_{k=n+1}^{\infty} \frac{1}{k^{r}} A_{k}\left(\theta, \widetilde{f}^{(r)}\right)= & \sum_{k=n+1}^{\infty}\left(\frac{1}{k^{r}}-\frac{1}{(k+1)^{r}}\right)\left[S_{k}\left(\theta, \widetilde{f}^{(r)}\right)-\widetilde{f}^{(r)}(\theta)\right] \\
& -\frac{1}{(n+1)^{r}}\left[S_{n}\left(\theta, \widetilde{f}^{(r)}\right)-\widetilde{f}^{(r)}(\theta)\right]
\end{aligned}
$$

by (16) we have

$$
\begin{aligned}
\left\|f-S_{n}(\cdot, f)\right\|_{L_{M}(\mathbb{T}, \omega) \leq} & \sum_{k=n+1}^{\infty}\left(\frac{1}{k^{r}}-\frac{1}{(k+1)^{r}}\right)\left\|S_{k}\left(\cdot, f^{(r)}\right)-f^{(r)}\right\|_{L_{M}(\mathbb{T}, \omega)} \\
& +\frac{1}{(n+1)^{r}}\left\|S_{n}\left(\cdot, f^{(r)}\right)-f^{(r)}\right\|_{L_{M}(\mathbb{T}, \omega)} \\
& +\sum_{k=n+1}^{\infty}\left(\frac{1}{k^{r}}-\frac{1}{(k+1)^{r}}\right)\left\|S_{k}\left(\cdot, \widetilde{f}^{(r)}\right)-\widetilde{f}^{(r)}\right\|_{L_{M}(\mathbb{T}, \omega)}
\end{aligned}
$$




$$
\begin{aligned}
& +\frac{1}{(n+1)^{r}}\left\|S_{n}\left(\cdot, \widetilde{f}^{(r)}\right)-\widetilde{f}^{(r)}\right\|_{L_{M}(\mathbb{T}, \omega)} \\
\leq & c_{1}\left\{\sum_{k=n+1}^{\infty}\left(\frac{1}{k^{r}}-\frac{1}{(k+1)^{r}}\right) E_{k}\left(f^{(r)}\right)_{M, \omega}+\frac{1}{(n+1)^{r}} E_{n}\left(f^{(r)}\right)_{M, \omega}\right\} \\
& +c_{2}\left\{\sum_{k=n+1}^{\infty}\left(\frac{1}{k^{r}}-\frac{1}{(k+1)^{r}}\right) E_{k}\left(\widetilde{f}^{(r)}\right)_{M, \omega}+\frac{1}{(n+1)^{r}} E_{n}\left(\widetilde{f}^{(r)}\right)_{M, \omega}\right\} .
\end{aligned}
$$

Since the sequence $\left\{E_{n}\left(f^{(r)}\right)_{M, \omega}\right\}$ is decreasing, using (16), we finally conclude that

$$
\begin{aligned}
& \left\|f-S_{n}(\cdot, f)\right\|_{L_{M}(\mathbb{T}, \omega)} \\
& \leq c_{1} E_{n}\left(f^{(r)}\right)_{M, \omega}\left\{\sum_{k=n+1}^{\infty}\left(\frac{1}{k^{r}}-\frac{1}{(k+1)^{r}}\right)+\frac{1}{(n+1)^{r}}\right\} \\
& \quad+c_{2} E_{n}\left(\tilde{f}^{(r)}\right)_{M, \omega}\left\{\sum_{k=n+1}^{\infty}\left(\frac{1}{k^{r}}-\frac{1}{(k+1)^{r}}\right)+\frac{1}{(n+1)^{r}}\right\} \\
& \leq c_{3} E_{n}\left(f^{(r)}\right)_{M, \omega}\left\{\sum_{k=n+1}^{\infty}\left(\frac{1}{k^{r}}-\frac{1}{(k+1)^{r}}\right)+\frac{1}{(n+1)^{r}}\right\} E_{n}\left(f^{(r)}\right)_{M, \omega} \\
& =\frac{2 c_{3}}{(n+1)^{r}} E_{n}\left(f^{(r)}\right)_{M, \omega} .
\end{aligned}
$$

Since $E_{n}(f)_{M, \omega} \leq\left\|f-S_{n}(\cdot, f)\right\|_{L_{M}(\mathbb{T}, \omega)}$, this gives (8) and completes the proof of Theorem 1 .

Corollary 6. For $f \in W_{M}^{r}(T, \omega)$ the inequality

$$
E_{n}(f)_{M, \omega} \leq \frac{c}{(n+1)^{r}}\left\|f^{(r)}\right\|_{L_{M}(\mathbb{T}, \omega)}
$$

holds with a constant $c$ independent of $n$.

Proof of Theorem 2. Let $\psi \in W_{M}^{2 k}(\mathbb{T}, \omega)$. Then by subadditivity of the best approximation and Corollary 6 , we have

$$
\begin{aligned}
E_{n}(f)_{M, \omega} & =E_{n}(f-\psi+\psi)_{M, \omega} \leq E_{n}(f-\psi)_{M, \omega}+E_{n}(\psi)_{M, \omega} \\
& \leq c\left\{\|f-\psi\|_{L_{M}(\mathbb{T}, \omega)}+\frac{1}{(n+1)^{2 k}}\left\|\psi^{(2 k)}\right\|_{L_{M}(\mathbb{T}, \omega)}\right\} .
\end{aligned}
$$

Since this inequality holds for every $\psi \in W_{M}^{2 k}(\mathbb{T}, \omega)$, by the definition of the $K$-functional we get

$$
E_{n}(f)_{M, \omega} \leq c K\left(\frac{1}{(n+1)^{2 k}}, f ; L_{M}(\mathbb{T}, \omega), W_{M}^{2 k}(\mathbb{T}, \omega)\right) .
$$


According to Theorem 8, this implies

$$
E_{n}(f)_{M, \omega} \leq c \Omega_{M, \omega}^{k}\left(\frac{1}{n+1}, f\right)
$$

which completes the proof.

Proof of Theorem 3. Let $\sum_{j=-\infty}^{\infty} \gamma_{j}(f) e^{i j x}$ be the exponential Fourier series of the boundary function of $f$, and $S_{n}(x, f)$ be its $n$th partial sum, i.e.,

$$
S_{n}(x, f)=\sum_{j=-n}^{n} \gamma_{j}(f) e^{i j x} .
$$

Then for $f \in H_{1}(\mathbb{D})$, by Theorem 3.4 in $[12]$ we have

$$
\gamma_{j}(f)= \begin{cases}a_{j}(f), & j \geq 0 \\ 0, & j<0\end{cases}
$$

Let $T_{n}^{*} \in \Pi_{n}$ be the polynomial of best approximation to $f \in L_{M}(\mathbb{T}, \omega)$. Then (15) and Theorem 2 for every natural number $n$ yield

$$
\begin{aligned}
\left\|f(z)-\sum_{j=0}^{n} a_{j}(f) z^{j}\right\|_{L_{M}(\mathbb{T}, \omega)}=\left\|f\left(e^{i x}\right)-\sum_{j=0}^{n} \gamma_{j}(f) e^{i j x}\right\|_{L_{M}(\mathbb{T}, \omega)} \\
\quad=\left\|f-S_{n}(\cdot, f)\right\|_{L_{M}(\mathbb{T}, \omega)}=\left\|f-T_{n}^{*}+T_{n}^{*}-S_{n}(\cdot, f)\right\|_{L_{M}(\mathbb{T}, \omega)} \\
\quad \leq\left\|f-T_{n}^{*}\right\|_{L_{M}(\mathbb{T}, \omega)}+\left\|S_{n}\left(\cdot, T_{n}^{*}-f\right)\right\|_{L_{M}(\mathbb{T}, \omega)} \\
\quad \leq c\left\|f-T_{n}^{*}\right\|_{L_{M}(\mathbb{T}, \omega)}=c E_{n}(f)_{M, \omega} \leq c \Omega_{M, \omega}^{k}\left(\frac{1}{n+1}, f\right) .
\end{aligned}
$$

Proof of Theorem 4. Let $f \in L_{M}(\mathbb{T}, \omega)$ and let $T_{n} \in \Pi_{n}(n=0,1,2, \ldots)$ be the polynomial of best approximation to $f$.

Let also $n$ be a natural number and $\delta:=1 / n$. By the subadditivity of $\Omega_{M, \omega}^{k}(\delta, \cdot)$,

$$
\Omega_{M, \omega}^{k}(\delta, f) \leq \Omega_{M, \omega}^{k}\left(\delta, f-T_{2^{j+1}}\right)+\Omega_{M, \omega}^{k}\left(\delta, T_{2^{j+1}}\right)
$$

for any $j=1,2, \ldots$, and by Corollary 3 ,

$$
\Omega_{M, \omega}^{k}\left(\delta, f-T_{2^{j+1}}\right) \leq c_{1}\left\|f-T_{2^{j+1}}\right\|_{L_{M}(T, \omega)}=c_{1} E_{2^{j+1}}(f)_{M, \omega} .
$$

Using Corollary 5 and Lemma 3 , and noting that the sequence $\left\{E_{n}(f)_{M, \omega}\right\}$ 
of best approximations is decreasing we get

$$
\begin{aligned}
& \Omega_{M, \omega}^{k}\left(\delta, T_{2^{j+1}}\right) \leq c_{2} \delta^{2 k}\left\|T_{2^{j+1}}^{(2 k)}\right\|_{L_{M}(\mathbb{T}, \omega)} \\
& \quad \leq c_{2} \delta^{2 k}\left\{\left\|T_{1}^{(2 k)}-T_{0}^{(2 k)}\right\|_{L_{M}(\mathbb{T}, \omega)}+\sum_{i=0}^{j}\left\|T_{2^{i+1}}^{(2 k)}-T_{2^{i}}^{(2 k)}\right\|_{L_{M}(\mathbb{T}, \omega)}\right\} \\
& \quad \leq c_{3} \delta^{2 k}\left\{\left\|T_{1}-T_{0}\right\|_{L_{M}(\mathbb{T}, \omega)}+\sum_{i=0}^{j} 2^{(i+1) 2 k}\left\|T_{2^{i+1}}-T_{2^{i}}\right\|_{L_{M}(\mathbb{T}, \omega)}\right\} \\
& \quad \leq c_{3} \delta^{2 k}\left\{E_{1}(f)_{M, \omega}+E_{0}(f)_{M, \omega}+\sum_{i=0}^{j} 2^{(i+1) 2 k}\left(E_{2^{i+1}}(f)_{M, \omega}+E_{2^{i}}(f)_{M, \omega}\right)\right\} \\
& \quad \leq c_{4} \delta^{2 k}\left\{E_{0}(f)_{M, \omega}+\sum_{i=0}^{j} 2^{(i+1) 2 k} E_{2^{i}}(f)_{M, \omega}\right\} \\
& \quad=c_{4} \delta^{2 k}\left\{E_{0}(f)_{M, \omega}+2^{2 k} E_{1}(f)_{M, \omega}+\sum_{i=1}^{j} 2^{(i+1) 2 k} E_{2^{i}}(f)_{M, \omega}\right\} .
\end{aligned}
$$

Since

$$
2^{(i+1) 2 k} E_{2^{i}}(f)_{M, \omega} \leq 2^{4 k} \sum_{m=2^{i-1}+1}^{2^{i}} m^{2 k-1} E_{m}(f)_{M, \omega}
$$

for $i \geq 1$, the last inequality yields

$$
\begin{aligned}
& \Omega_{M, \omega}^{k}\left(\delta, T_{2^{m+1}}\right) \\
& \quad \leq c_{4} \delta^{2 k}\left\{E_{0}(f)_{M, \omega}+2^{2 k} E_{1}(f)_{M, \omega}+2^{4 k} \sum_{m=2}^{2^{j}} m^{2 r-1} E_{m}(f)_{M, \omega}\right\} \\
& \quad \leq c_{5} \delta^{2 k}\left\{E_{0}(f)_{M, \omega}+\sum_{m=1}^{2^{j}} m^{2 r-1} E_{m}(f)_{M, \omega}\right\} .
\end{aligned}
$$

Selecting $j$ such that $2^{j} \leq n<2^{j+1}$, from (23) we have

$$
\begin{aligned}
E_{2^{j+1}}(f)_{M, \omega} & =\frac{2^{(j+1) 2 k} E_{2^{j+1}}(f)_{M, \omega}}{2^{(j+1) 2 k}} \leq \frac{2^{(j+1) 2 k} E_{2^{j}}(f)_{M, \omega}}{n^{2 k}} \\
& \leq \frac{2^{4 k}}{n^{2 k}} \sum_{m=2^{j-1}+1}^{2^{j}} m^{2 k-1} E_{m}(f)_{M, \omega} \cdot
\end{aligned}
$$

Now combining (21), (22), (24) and the last relation we obtain the inequality (11) of Theorem 4. 
Proof of Theorem 5. For the polynomials of best approximation we obtain

$$
\begin{aligned}
\left\|T_{2^{i+1}}-T_{2^{i}}\right\|_{L_{M}(\mathbb{T}, \omega)} & \leq\left\|T_{2^{i+1}}-f\right\|_{L_{M}(\mathbb{T}, \omega)}+\left\|f-T_{2^{i}}\right\|_{L_{M}(\mathbb{T}, \omega)} \\
& =E_{2^{i+1}}(f)_{M, \omega}+E_{2^{i}}(f)_{M, \omega} \\
& \leq 2 E_{2^{i}}(f)_{M, \omega} \leq 2^{(i+1) 2 r} E_{2^{i}}(f)_{M, \omega}
\end{aligned}
$$

and hence using the Bernstein inequality (14) we have

$$
\begin{aligned}
\left\|T_{2^{i+1}}^{(2 r)}-T_{2^{i}}^{(2 r)}\right\|_{L_{M}(\mathbb{T}, \omega)} & \leq c_{6} 2^{(i+1) 2 r}\left\|T_{2^{i+1}}-T_{2^{i}}\right\|_{L_{M}(\mathbb{T}, \omega)} \\
& \leq c_{7} 2^{(i+1) 2 r} E_{2^{i}}(f)_{M, \omega}
\end{aligned}
$$

Now recalling the definition $(7)$ of the norm in $W_{M}^{2 r}(\mathbb{T}, \omega)$ and by $(25)$, (26) and (23) we get

$$
\begin{aligned}
\sum_{i=1}^{\infty} \| T_{2^{i+1}} & -T_{2^{i}} \|_{W_{M}^{2 r}(\mathbb{T}, \omega)} \\
& =\sum_{i=1}^{\infty}\left\|T_{2^{i+1}}-T_{2^{i}}\right\|_{L_{M}(\mathbb{T}, \omega)}+\sum_{i=1}^{\infty}\left\|T_{2^{2+1}}^{(2 r)}-T_{2^{i}}^{(2 r)}\right\|_{W_{M}^{2 r}(\mathbb{T}, \omega)} \\
& \leq \sum_{i=1}^{\infty} 2^{(i+1) 2 r} E_{2^{i}}(f)_{M, \omega}+c_{7} \sum_{i=1}^{\infty} 2^{(i+1) 2 r} E_{2^{i}}(f)_{M, \omega} \\
& =c_{8} \sum_{i=1}^{\infty} 2^{(i+1) 2 r} E_{2^{i}}(f)_{M, \omega} \leq c_{8} 2^{4 r} \sum_{i=1}^{\infty}\left(\sum_{m=2^{i-1}+1}^{2^{i}} m^{2 r-1} E_{m}(f)_{M, \omega}\right) \\
& =c_{9} \sum_{m=2}^{\infty} m^{2 r-1} E_{m}(f)_{M, \omega},
\end{aligned}
$$

which by the condition (12) of Theorem 5 implies that

$$
\sum_{i=1}^{\infty}\left\|T_{2^{i+1}}-T_{2^{i}}\right\|_{W_{M}^{2 r}(\mathbb{T}, \omega)}<\infty,
$$

and hence

$$
\left\|T_{2^{i+1}}-T_{2^{i}}\right\|_{W_{M}^{2 r}(\mathbb{T}, \omega)} \rightarrow 0 \quad \text { as } n \rightarrow \infty .
$$

This means that $\left\{T_{2^{i}}\right\}$ is a Cauchy sequence in $W_{M}^{2 r}(\mathbb{T}, \omega)$. Since $T_{2^{i}} \rightarrow f$ in $L_{M}(\mathbb{T}, \omega)$ and $W_{M}^{2 r}(\mathbb{T}, \omega)$ is a Banach space we have $f \in W_{M}^{2 r}(\mathbb{T}, \omega)$.

Acknowledgements. The authors are indebted to the referees for valuable suggestions.

\section{References}

[1] N. K. Bary, A Treatise on Trigonometric Series, Vol. I, Pergamon Press, 1964. 
[2] C. Bennett and R. Sharpley, Interpolation of Operators, Academic Press, 1988.

[3] A. Böttcher and Yu. I. Karlovich, Carleson Curves, Muckenhoupt Weights, and Toeplitz Operators, Birkhäuser, 1997.

[4] D. W. Boyd, Spaces between a pair of reflexive Lebesgue spaces, Proc. Amer. Math. Soc. 18 (1967), 215-219.

[5] -, Indices of function spaces and their relationship to interpolation, Canad. J. Math. 21 (1969), 1245-1254.

[6] —, Indices for the Orlicz spaces, Pacific J. Math. 38 (1971), 315-323.

[7] M. C. De Bonis, G. Mastroianni and M. G. Russo, Polynomial approximation with special doubling weights, Acta Sci. Math. (Szeged) 69 (2003), 159-184.

[8] R. A. De Vore and G. G. Lorentz, Constructive Approximation, Springer, 1993.

[9] Z. Ditzian and V. Totik, K-functionals and best polynomial approximation in weighted $L^{p}(R)$, J. Approx. Theory 46 (1986), 38-41.

[10] - - - K-functionals and weighted moduli of smoothness, ibid. 63 (1990), 3-29.

[11] - - - Moduli of Smoothness, Springer Ser. Comput. Math. 9, Springer, New York, 1987.

[12] P. L. Duren, Theory of $H^{p}$ Spaces, Academic Press, 1970.

[13] E. M. Dynkin and B. P. Osilenker, Weighted norm estimates for singular integrals and their applications, Itogi Nauki i Tekhniki Ser. Mat. Anal. 21 (1983), 42-129 (in Russian); English transl.: J. Soviet Math. 30 (1985), 2094-2154.

[14] G. Freud and H. N. Mhaskar, Weighted polynomial approximation in rearrangement invariant Banach function spaces on the whole real line, Indian J. Math. 22 (1980), 209-294.

[15] - - - K-functionals and moduli of continuity in weighted polynomial approximation, Ark. Mat. 21 (1983), 145-161.

[16] E. A. Haciyeva, Investigation of the properties of functions with quasimonotone Fourier coefficients in generalized Nikol'skiu-Besov spaces, author's summary of a dissertation, Tbilisi, 1986 (in Russian).

[17] R. Hunt, B. Muckenhoupt and R. Wheeden, Weighted norm inequalities for the conjugate function and Hilbert transform, Trans. Amer. Math. Soc. 176 (1973), $227-251$.

[18] R. A. Hunt and W. Young, A weighted norm inequality for Fourier series, Bull. Amer. Math. Soc. 80 (1974), 274-277.

[19] D. M. Israfilov, Approximation by p-Faber polynomials in the weighted Smirnov class $E^{p}(G, \omega)$ and the Bieberbach polynomials, Constr. Approx. 17 (2001), 335-351.

[20] - , Approximation by $p$-Faber-Laurent rational functions in the weighted Lebesgue spaces, Czechoslovak Math. J. 54 (129) (2004), 751-765.

[21] D. M. Israfilov and A. Guven, Approximation in weighted Smirnov classes, East J. Approx. 11 (2005), 1-12.

[22] A. Yu. Karlovich, Algebras of singular integral operators with piecewise continuous coefficients on reflexive Orlicz spaces, Math. Nachr. 179 (1996), 187-222.

[23] - On the essential norm of the Cauchy singular operator in weighted rearrangement-invariant spaces, Integral Equations Operator Theory 38 (2000), 28-50.

[24] - Algebras of singular integral operators with PC coefficients in rearrangement invariant spaces with Muckenhoupt weights, J. Operator Theory 47 (2002), 303-323.

[25] —, Fredholmness of singular integral operators with piecewise continuous coefficients on weighted Banach function spaces, J. Integral Equations Appl. 15 (2003), 263-320.

[26] M. A. Krasnosel'skiı̆ and Ya. B. Rutickiŭ, Convex Functions and Orlicz Spaces, Noordhoff, 1961.

[27] N. X. Ky, On approximation by trigonometric polynomials in $L_{u}^{p}$-spaces, Studia Sci. Math. Hungar. 28 (1993), 183-188. 
[28] L. Maligranda, Indices and interpolation, Dissertationes Math. 234 (1985).

[29] G. Mastroianni and V. Totik, Weighted polynomial inequalities with doubling and $A_{\infty}$ weights, Constr. Approx. 16 (2000), 37-71.

[30] -, 一, Jackson type inequalities for doubling and $A_{p}$ weights, in: Proc. Third International Conference on Functional Analysis and Approximation Theory, Vol. 1 (Acquafredda di Maratea, 1996), Rend. Circ. Mat. Palermo (2) Suppl. 52, Vol. 1 (1998), 83-99.

[31] - - - Best approximation and moduli of smoothness for doubling weights, J. Approx. Theory 110 (2001), 180-199.

[32] W. Matuszewska and W. Orlicz, On certain properties of $\varphi$-functions, Bull. Acad. Polon. Sci. Sér. Sci. Math. Astronom. Phys. 8 (1960), 439-443.

[33] H. N. Mhaskar, Introduction to the Theory of Weighted Polynomial Approximation, Series in Approximation and Decompositions 7, World Sci., River Edge, NJ, 1996.

[34] H. N. Mhaskar and E. B. Saff, Where does the $L^{p}$-norm of a weighted polynomial live?, Trans. Amer. Math. Soc. 303 (1987), 37-71.

[35] B. Muckenhoupt, Weighted norm inequalities for the Hardy maximal function, ibid. 165 (1972), 207-226.

[36] A.-R. K. Ramazanov, On approximation by polynomials and rational functions in Orlicz Spaces, Anal. Math. 10 (1984), 117-132.

[37] M. M. Rao and Z. D. Ren, Theory of Orlicz Spaces, Dekker, 1991.

[38] M. Rosenblum, Summability of Fourier series in $L^{p}(d \mu)$, Trans. Amer. Math. Soc. 165 (1962), 32-42.

[39] K. Runovski, On Jackson type inequality in Orlicz classes, Rev. Mat. Complut. 14 (2001), 395-404.

[40] A. F. Timan, Theory of Approximaton of Functions of a Real Variable, Pergamon Press and MacMillan, 1963; Russian original published by Fizmatgiz, Moscow, 1960.

[41] G. Wu, On approximation by polynomials in Orlicz spaces, Appox. Theory Appl. 7 (1991), no. 3, 97-110.

Department of Mathematics

Faculty of Art and Science

Balikesir University

10145 Balikesir, Turkey

E-mail: mdaniyal@balikesir.edu.tr

aguven@balikesir.edu.tr

Received February 7, 2005

(5577) 